\title{
Localized Jacobian ILU preconditioners for hydraulic fractures
}

\author{
A. P. Peirce ${ }^{* \dagger}$ \\ Department of Mathematics, University of British Columbia, Vancouver, BC, Canada V6T 1Z2
}

\begin{abstract}
SUMMARY
We discuss the properties of a class of sparse localized approximations to the Jacobian operator that arises in modelling the evolution of a hydraulically driven fracture in a multi-layered elastic medium. The governing equations involve a highly non-linear coupled system of integro-partial differential equations along with the fracture front free boundary problem. We demonstrate that an incomplete LU factorization of these localized Jacobians yields an efficient preconditioner for the fully populated, stiff, non-symmetric system of algebraic equations that need to be solved multiple times for every growth increment of the fracture. The performance characteristics of this class of preconditioners is explored via spectral analysis and numerical experiment. Copyright (C) 2005 John Wiley \& Sons, Ltd.
\end{abstract}

KEY WORDS: ILU preconditioning; hydraulic fracture; BEM

\section{INTRODUCTION}

Hydraulic fracturing is a process by which a fracture is propagated in brittle rock by injecting a viscous fluid into a perforated section of a bore-hole under a sufficiently high pressure to overcome the tensile strength of the rock and the far-field minimum principal geological stresses. As a result, a fracture surface, which is often assumed to be planar, develops in a direction perpendicular to this minimum principal stress. Hydraulic fracturing is frequently used in the oil and gas recovery industry to induce fractures in reservoirs in order to substantially enhance the flow of hydrocarbons. The process has also been used in the mining industry to introduce large fractures in the rock surrounding mining excavations in order to enlarge these excavations without having to use explosive charges. Environmental engineers have also used hydraulic fracturing to isolate toxic substances by injecting impermeable materials into fractures. In all these processes, it is desirable to be able to predict the evolution of the fracture surface

\footnotetext{
*Correspondence to: A. P. Peirce, Department of Mathematics, University of British Columbia, Vancouver, BC, Canada V6T 122 .

†E-mail: peirce@math.ubc.ca

Contract/grant sponsor: Department of Mathematics, University of British Columbia Contract/grant sponsor: NSERC
}

Copyright (c) 2005 John Wiley \& Sons, Ltd.

Received 3 January 2005

Revised 9 June 2005

Accepted 9 August 2005 
under known stress and geological conditions. Thus robust, efficient, and accurate numerical modelling of hydraulically driven fractures is of considerable interest.

The governing equations that describe the evolution of a hydraulic fracture involve: the twodimensional (2D) Reynold's lubrication equation expressing the conservation of fluid volume within the planar fracture; the three-dimensional (3D) equilibrium and elastic stress-strain partial differential equations that in this case can be reduced to a (2D) boundary integral equation expressing the balance of forces between the fluid pressure, the geological stresses, and the elastic response of the rockmass; and a propagation criterion which determines the extent of the fracture footprint. Previous papers (see for example References [1-4] have described the governing equations and the appropriate discretization procedures for the efficient numerical modelling of hydraulic fractures. In this paper, we are concerned with the efficient solution of the stiff system of non-linear equations that need to be solved at each time step in order to determine the fracture width and fluid pressure fields within the fracture. Application of Newton's method reduces this problem to the solution of a large system of fully populated non-symmetric linear algebraic equations. In a previous paper [5] we described a dual mesh multigrid preconditioner for the efficient solution of these equations. In that paper a localized approximation to the Jacobian, when combined with Gauss-Seidel iteration, was found to provide a very efficient smoother of the high-frequency modes of the fully populated Jacobian matrix. In this paper we explore the efficacy of preconditioners based on incomplete LU factorizations of a class of localized Jacobians that are an extension of the one introduced as a smoother in Reference [5]. In particular, we investigate some of the spectral properties of the localized Jacobians for a one-dimensional (1D) model problem. This analysis indicates that these localized Jacobian operators provide efficient preconditioners for the fully populated Jacobians. For 2D problems we demonstrate that zero fill incomplete LU factorizations of the localized Jacobian operators yield efficient preconditioners for the fully populated system.

In Section 2, we describe the continuous and discrete coupled integro-partial differential equations that govern the evolution of a fluid-driven fracture. In Section 3, we describe the class of localized Jacobian operators, present an analysis of their spectral properties for a 1D model problem, and discuss the spectral properties of the localized Jacobians for 2D problems as well as the spectra of the ILU factorizations of the localized Jacobians. In Section 4, we present results on the performance of preconditioners based on the ILU factorizations of the localized Jacobians in a numerical example. In Section 5 we make some concluding remarks.

\section{GOVERNING EQUATIONS AND DISCRETIZATION}

In a three-dimensional (3D) layered elastic medium the integral equation governing the width profile for a planar crack can be written in the form

$$
C w=\int_{\Omega(t)} C(x, y ; \xi, \eta) w(\xi, \eta, t) \mathrm{d} \xi \mathrm{d} \eta=p(x, y, t)-\sigma_{c}(x, y)
$$

where the fracture is subjected to a fluid pressure $p(x, y, t)$ that works against the far-field minimum principal stress field $\sigma_{c}(x, y)$ (also known as the confining stress) within the elastic medium. For example, in the case of an elastic material with zero toughness, if $p$ is sufficiently large to overcome $\sigma_{c}$ so that the net pressure $p(x, y, t)-\sigma_{c}(x, y)$ is positive, then the fracture opens by an amount $w(\xi, \eta, t)>0$. Due to the non-locality of the operator $C$ it is possible 
for $w$ to be positive at points where the net pressure is negative. However, if the net pressure is sufficiently negative at some point then $w<0$, which would imply that the sides of the fracture surface inter-penetrate. At these so-called 'pinch-points' it is necessary to impose some form of minimum width constraint $w \geqslant w_{c}$. The Green's function $C(x, y ; \xi, \eta)$ contains all the information about the compliance of the layered elastic medium. The fracture at time $t$ is assumed to occupy the region denoted by $\Omega(t)$, which has a boundary that we denote by $\partial \Omega$. A procedure for constructing the Green's functions for layered elastic materials both in plane strain and in three dimensions is described in Reference [3].

In the case of a planar fracture that grows in a 3D elastic medium, the fluid flow equations are well approximated by the 2D Reynold's lubrication equation:

$$
\frac{\partial w}{\partial t}=\nabla \cdot(D(w,|\nabla p|) \nabla p)+\delta(x, y) Q
$$

where $Q=Q(x, y, t)$ is the volume of fluid pumped into the fracture at the well bore. For simplicity, we have assumed that the surrounding rockmass is impermeable so that there is no leakoff of the fluid within the fracture into the surrounding elastic medium. If the fluid is Newtonian $D(w)=w^{3} / 12 \mu$, where $\mu$ is the fluid viscosity, while for a non-Newtonian power-law fluid $D(w)$ is replaced by $D(w,|\nabla p|)$ in which the dependence of $D$ on $w$ is larger than a cubic power while $D$ has a power-law dependence on $|\nabla p|$ in which the exponent is positive.

We assume that the fracture region $\Omega(t)$ evolves in a window that has been divided into rectangular elements. For the discretization of the elastic integral equation (1) we assume that the width is piece-wise constant over each element and collocate at the element centres. In this case the discrete elasticity equations assume the form

$$
C w=p-\sigma_{c}
$$

In the case of an inhomogeneous elastic medium the discretized collocation operator $C$ is not symmetric.

By integrating both sides of (2) over a control element $\Delta x \Delta y$ (see Reference [5]) and assuming that $w$ and $p$ are piecewise constant over each cell, the resulting set of difference equations can be expressed in the form

$$
\frac{\partial w}{\partial t}=A(w) p+F
$$

Substituting the pressure from (3) into (4) we obtain a non-linear evolution equation for $w$ :

$$
\frac{\partial w}{\partial t}=A(w) C w+F
$$

By linearizing a 1D version of this evolution equation and applying Fourier analysis, it can be shown (see (10) and (11) below) that the eigenvalues of the system matrix $A C$ are given by

$$
\widehat{[A C}]_{k}=-\frac{2 E^{\prime} \bar{D}}{\Delta x^{3}} \sin ^{3}\left(\frac{|k| \Delta x}{2}\right)
$$

Therefore an explicit time stepping scheme will be subject to a time step restriction in which $\Delta t<K \Delta x^{3}$. Because the operator $C$ is fully populated, this small time step restriction renders 
explicit time stepping impracticable. Due to the stiffness of this evolution equation we will assume in the remainder of this paper that the backward Euler scheme is used to march the solution forward in time.

Our objective is to investigate efficient algorithms to solve the large system of non-linear algebraic equations that need to be solved at each time step. We use Newton's method to solve for the width $w$ within the crack surface at the end of each time step, which can be expressed in the form

$$
J(A C) \delta w_{k}=-r_{k}
$$

where $r_{k}=\Delta w_{k}-\Delta t A\left(w_{k}\right) C w_{k}-\Delta t F$ is the $k$ th residual. Since $C$ is not a symmetric matrix, the Jacobian $J(A C)$ is also not symmetric. Pinch points, at which width constraints are active, typically have an adverse effect on the conditioning of these equations. For this reason the numerical example we have chosen to illustrate the performance of the preconditioners has a significant number of pinch point elements.

\section{LOCALIZED JACOBIANS AND SPECTRAL PROPERTIES}

\subsection{Localized Jacobian operators}

In order to improve the conditioning of the linear system (7), we consider a class (which we refer to as class I) of sparse approximations to the Jacobian, which are constructed by exploiting the sparseness of the fluid flow operator $A(w)$ and the rapid $O\left(1 / r^{3}\right)$ spatial decay of the elements of the elasticity operator $C$. The construction of the localized Jacobians is depicted in Figure 1(a). Consider the receiving point $(i, j)$ at the centre of a $5 \times 5$ patch of elements. The crucial component in this process is the approximation of the matrix product $A C$. We observe that, due to the local support of the matrix $A$, the receiving point $(i, j)$ is only influenced by the pressures at the neighbouring points $(i, j+1),(i, j-1),(i-1, j)$, $(i+1, j)$ as well the pressure at the point $(i, j)$. These pressure points are indicated by the solid circles $(\bullet)$ in Figure 1(a). Since $C$ is fully populated, the pressures at each of these neighbouring points depends on the widths throughout the current fracture surface. However, because the coefficients of the $C$ matrix decay at a rate of $O\left(1 / r^{3}\right)$ as the distance $r$ from the sending element increases, we adopt an approximation that only includes the elastic influences due to the widths in each of the nearest neighbours to the pressure points (•). In Figure 1(a) we depict by grey shading the set of $3 \times 3$ nearest neighbour width elements that are used to approximate the pressure at the point $(i, j+1)$, while the $C$ matrix influences due to the widths at all remaining elements within the fracture are ignored. Similar patches of $3 \times 3$ elements are constructed around each of the five elements of the difference stencil for $A$ that are denoted by the solid circles. The matrix vector product can be expressed in the form

$$
(A C)_{m n}=\sum_{k \in \mathscr{N}_{M}^{5}} A_{m k} C_{k n} \approx \begin{cases}\sum_{k \in \mathscr{N}_{m}^{5}} A_{m k} C_{k n} & \text { when } n \in \mathscr{N}_{k}^{9} \\ 0 & \text { when } n \notin \mathscr{N}_{k}^{9}\end{cases}
$$

Here we have used the indices $k, m$, and $n$ to denote a global scheme to number the elements as opposed to the co-ordinate based scheme depicted in Figure 1(a). The receiving element 


\begin{tabular}{|c|c|c|c|c|}
\hline 0 & 0 & 0 & 0 & 0 \\
\hline 0 & $\stackrel{\circ}{\mathrm{O}-1, j+1}$ & $\stackrel{\bullet}{i, j+1}$ & $\stackrel{\circ}{i+1, j+1}$ & 0 \\
\hline 0 & $\mathrm{j}-1, \mathrm{j}$ & $\stackrel{\bullet}{\mathrm{i}, \mathrm{j}}$ & $\stackrel{\bullet}{\mathrm{i}+1, \mathrm{j}}$ & 0 \\
\hline 0 & $\stackrel{\circ}{\mathrm{i}-1, \mathrm{j}-1}$ & $\mathrm{i}, \dot{\mathrm{j}}-1$ & $\stackrel{\circ}{i+1, j-1}$ & 0 \\
\hline 0 & 0 & 0 & 0 & 0 \\
\hline
\end{tabular}

(a)

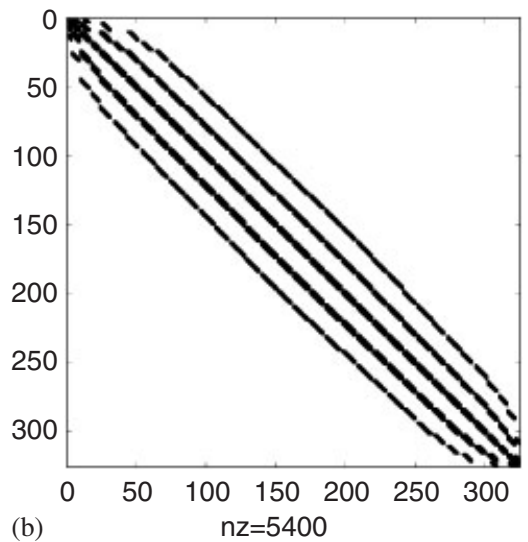

(b)

Figure 1. (a) Geometric view of the procedure used to construct the elements of the localized Jacobian associated with node $(i, j)$; and (b) the sparsity pattern for the localized Jacobian obtained by discarding all but the nearest-neighbour elasticity influences.

$(i, j)$ in this scheme corresponds to the element number $m$. Here $\mathscr{N}_{m}^{5}$ represents the five elements associated with the difference stencil of $A$ centred at element $m$, while $\mathscr{N}_{k}^{9}$ refers to the $k$ th stencil element as well as its eight neighbours-such as those shaded elements that are associated with the stencil element $(i, j+1)$. Following the procedure described above, the fully populated Jacobian is approximated by a sparse localized Jacobian that has at most 21 non-zero elements in each row, which implies that the number of non-zero elements in the localized Jacobian is a factor of $21 / N$ smaller than that of the full Jacobian-we will refer to this ratio as the fill factor. In Figure 1(b) the non-zero elements in the localized Jacobian are plotted. In what follows we will denote this localized Jacobian by $J_{3 \times 3}$, because of the $3 \times 3$ patch comprising the nearest neighbours and the receiving pressure point itself. Because $J_{3 \times 3}$ only includes the nearest neighbour influences of $C$, we can expect that it will approximate the high-frequency components of the spectrum of $J$ relatively accurately while it will yield a poor approximation of the low frequency components that are associated with the long range influences in $C$ that were discarded.

There are a number of extensions to this procedure for constructing the localized Jacobian described above. For example, we could construct $J_{5 \times 5}$ by incorporating the next-to-nearestneighbour influences as well as the nearest-neighbour influences. In this case $\mathscr{N}_{k}^{9}$ in (8) is replaced by $\mathscr{N}_{k}^{25}$ comprising the $5 \times 5$ patch of neighbours around each pressure point. We can expect that $J_{5 \times 5}$ captures more of the low-frequency spectrum than $J_{3 \times 3}$ but has more non-zero elements (the fill factor is increased from $21 / N$ to $45 / N$ ), which is more expensive to store and involves more CPU time to implement the ILU factorization. Since $J_{5 \times 5}$ is a direct extension of $J_{3 \times 3}$ to next-nearest-neighbours it is also regarded as a member of class I. In order to incorporate more of the influence coefficients from the $C$ operator while preserving the same sparsity structure for the localized Jacobian, we consider another class (class II) of localized Jacobians. To construct these, we include, in the approximate evaluation of the pressures at the five pressure points $(\bullet)$, all the elastic influences from the 21 elements of the $5 \times 5$ patch of elements (excluding the influences of only the corner elements $(i \pm 2, j \pm 2$ ) 
of the $5 \times 5$ patch). In this way we obtain a localized Jacobian that has precisely the same number of non-zero elements as $J_{3 \times 3}$, but which includes more of the elements of $C$ in its construction.

\subsection{Spectral analysis of the localized Jacobians for a model problem}

In order to analyse the efficacy of the localized Jacobian as a preconditioner, we consider a simple 1D model problem comprising a symmetric fracture propagating in a situation of plane strain. In this case the governing integral equation is given by

$$
C w=-\frac{E^{\prime}}{4 \pi} \int_{-l(t)}^{l(t)} \frac{w(s, t)}{(s-x)^{2}} \mathrm{~d} s=p(x, t)-\sigma_{c}(x)
$$

where $l(t)$ is the half-length of the fracture and $E^{\prime}=E /\left(1-v^{2}\right)$. Assuming piecewise constant basis functions and collocation at element centres, we obtain the following discrete form of the integral equation:

$$
\sum_{n=1}^{N} C_{m n} w_{n}=p_{m}-\left(\sigma_{c}\right)_{m}, \quad C_{m n}=-\frac{E^{\prime}}{4 \pi \Delta x}\left[\frac{1}{(m-n)^{2}-\frac{1}{4}}\right]
$$

The applicable Reynold's equation in the absence of leakoff is given by

$$
\frac{\partial w}{\partial t}=\frac{\partial}{\partial x}\left(D(w) \frac{\partial p}{\partial x}\right)+\delta(x) Q=A p+\delta(x) Q
$$

For the purposes of this analysis, we assume that the width is slowly varying and frozen to some nominal value $\bar{w}$, that $\bar{D}:=D(\bar{w})$, and that the discrete form of the $A$ operator is

$$
A p_{n}=\frac{\bar{D}}{\Delta x^{2}}\left(p_{n+1}-2 p_{n}+p_{n-1}\right)
$$

Because of the Toeplitz form of these discrete equations, the discrete Fourier transform of the kernel function yields the eigenvalues of the combined discrete operator $A C$ which are given by (6).

Combining (10) and (11) the elements of the discrete matrix product $A C$ can be expressed in the form:

$$
[A C]_{n-j, n}= \begin{cases}-\frac{\bar{D} E^{\prime}}{4 \pi \Delta x^{3}} \frac{3 \cdot 2^{5}}{(2 j-3)(2 j-1)(2 j+1)(2 j+3)} & \text { if } j \geqslant 2 \\ \frac{\bar{D} E^{\prime}}{4 \pi \Delta x^{3}} \frac{2^{5} \cdot 3}{5 \cdot 3} & \text { if } j=1 \\ -\frac{\bar{D} E^{\prime}}{4 \pi \Delta x^{3}} \frac{2^{5} \cdot 3}{3 \cdot 3} & \text { if } j=0\end{cases}
$$

The class I localized operators that are formed by truncating $C_{m n}$ beyond $L$ neighbours is represented by $J_{2 L+1}=J\left(A C_{2 L+1}\right)$. By taking the Fourier transform of $A C_{2 L+1}$ we obtain 

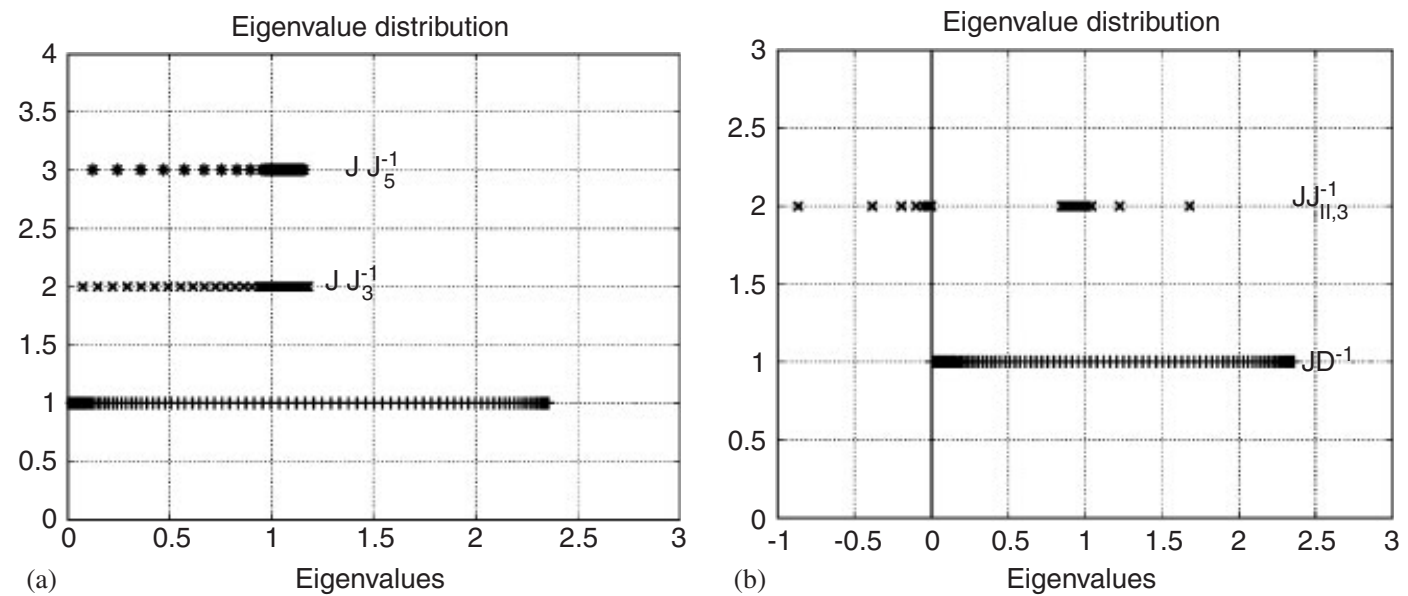

Figure 2. (a) The clustering of the eigenvalues of the model problem due to preconditioning by the localized Jacobians can be seen by comparing the spectra of $J D^{-1}, J J_{3}^{-1}$, and $J J_{5}^{-1}$; and (b) the spectra of $J D^{-1}$ and $J J_{\mathrm{II}, 3}^{-1}$ demonstrate that the class II localized Jacobains introduce significant clustering, but are unsuitable as preconditioners as the conditioned problem is indefinite.

the following expression for the eigenvalues of the localized discrete operator:

$$
\begin{aligned}
{\left[\widehat{A C_{2 L+1}}\right]_{k} } & =-2^{5} \cdot 3 \frac{\bar{D} E^{\prime}}{4 \pi \Delta x^{3}}\left\{\frac{1}{3^{2}}-\frac{2}{5 \cdot 3} \cos k \Delta x+2 \sum_{l=2}^{L} \frac{\cos k l \Delta x}{(2 l-3)(2 l-1)(2 l+1)(2 l+3)}\right\} \\
& =-\frac{4 \bar{D} E^{\prime}}{\pi \Delta x^{3}} \sin ^{2}\left(\frac{|k| \Delta x}{2}\right)\left\{1-2 \sum_{l=1}^{L} \frac{\cos k l \Delta x}{4 l^{2}-1}\right\}
\end{aligned}
$$

For the class II operators we only present the case of the nearest-neighbour localized Jacobian. By performing an analogous calculation to that above, the eigenvalues of the localized operator $A C_{\mathrm{II}, 3}$ of the class II localized Jacobian $J_{\mathrm{II}, 3}$ can be shown to be

$$
\widehat{A C_{\mathrm{II}, 3}}=-\frac{8 \bar{D} E^{\prime}}{\pi \Delta x^{3}}\left\{\frac{1}{3}-\frac{2}{5} \cos k \Delta x+\frac{2}{35} \cos 2 k \Delta x\right\}
$$

By taking the quotient of the spectra for the full Jacobian (6) and that of the localized Jacobians $J_{3}$ and $J_{5}$ (12), we obtain the spectra of the preconditioned Jacobian, which are plotted in Figure 2(a). In this figure the clustering of the eigenvalues of $J J_{3}^{-1}$, and $J J_{5}^{-1}$ compared to those of $J D^{-1}$ can be clearly observed. In Figure 2(b) the spectra of $J D^{-1}$ and $J J_{\mathrm{II}, 3}^{-1}$ are compared. From this figure it is evident that preconditioning by this localized Jacobian $J_{\text {II } 3}^{-1}$ can be expected to introduce significant clustering of the system eigenvalues, but it is unsuitable as a preconditioner since the conditioned problem is indefinite. For this reason the class II preconditioners are not investigated further in this paper. 


\subsection{Spectral properties of the localized Jacobian for planar fractures}

Based on the results of the model problem analysis, there is reason to expect that the class I localized Jacobians $J_{n \times n}$ should provide efficient preconditioners. In this section we investigate the spectral properties of these preconditioners for a planar hydraulic fracture comprising $N=325$ elements involving a confining stress jump situation as described in the example presented in Section 4. In Figure 3(a) and (b) we provide the results for localized Jacobians $J_{3 \times 3}$ and $J_{5 \times 5}$, respectively. In these two figures the $\bullet$ symbols represent $\log _{10}\left|\operatorname{eig}\left(J J_{n \times n}^{-1}-I\right)\right|$, the symbols represent $\log _{10}\left|\operatorname{eig}\left(J\left(L_{0} U_{0}\right)_{n \times n}^{-1}-I\right)\right|$, and the $\star$ symbols represent $\log _{10} \mid \operatorname{eig}\left(J D^{-1}-\right.$ $I) \mid$. Here $J_{n \times n}^{-1}$ is the inverse matrix of the localized Jacobian and represents the limit of the advantage to be gained from some form of incomplete factorization, while $D^{-1}$ is the inverse of the diagonal matrix of $J$, which represents some minimal form of preconditioning. Here $\left(L_{0} U_{0}\right)_{n \times n} \approx J_{n \times n}$ represents the $I L U(0)$ factorization (see References $[6,7]$ ) with zero fill, which has been chosen above more complete factorizations, $I L U(k)$ with $k>0$, in order to minimize the additional memory resources required for implementation. We observe that preconditioning by $J_{n \times n}^{-1}$ achieves a substantial clustering of the eigenvalues of $J J_{n \times n}^{-1}$ around 1. Indeed, for the $3 \times 3$ case all but a few modes can be damped by a factor of $10^{-1}$ in each iteration, while for the $5 \times 5$ case this is even more pronounced. For both $\left(L_{0} U_{0}\right)_{3 \times 3}^{-1}$ and $\left(L_{0} U_{0}\right)_{5 \times 5}^{-1}$ the $I L U(0)$ factorizations manage to achieve substantial clustering too. For $\left(L_{0} U_{0}\right)_{3 \times 3}^{-1}$ only 8 modes are more than a distance $10^{-1}$ from 1 , while for $\left(L_{0} U_{0}\right)_{5 \times 5}^{-1}$ only two modes are more than a distance of $10^{-1}$ from 1 . In order to observe how this clustering translates into improved performance, in the legend of Figure 3 we have provided the iteration counts
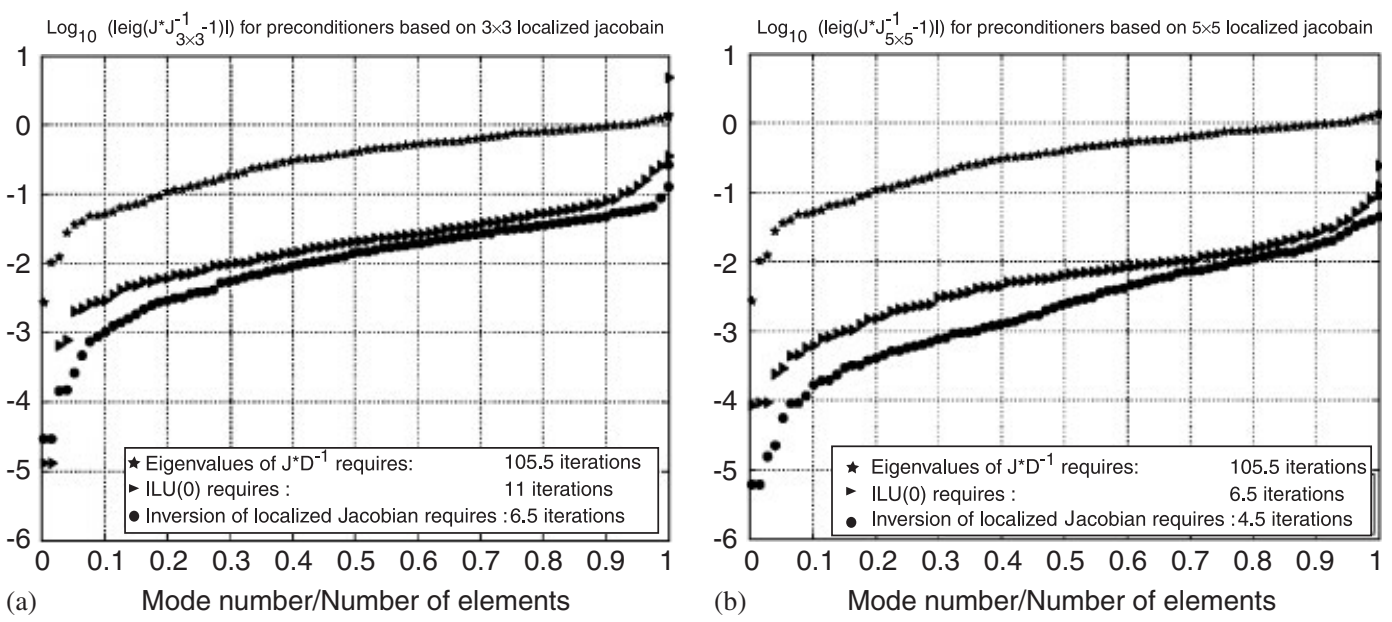

Figure 3. (a) A semi- $\log _{10}$ plot of the absolute values of the eigenvalues of $J D^{-1}-I$, $J\left(L_{0} U_{0}\right)_{3 \times 3}^{-1}-I$, and $J J_{3 \times 3}^{-1}-I$. These give a measure of the extent to which the modes can be damped in each iterate. The legend gives iterative performance of the preconditioner combined with a BiCGSTAB solver; and (b) a semi- $\log _{10}$ plot of the absolute values of the eigenvalues of $J D^{-1}-I, J\left(L_{0} U_{0}\right)_{5 \times 5}^{-1}-I$, and $J J_{5 \times 5}^{-1}-I$. The legend gives iterative performance of the preconditioner combined with a BiCGSTAB solver. 
when these preconditioners are used in conjunction with the BiCGSTAB algorithm (see References $[8,9])$. The preconditioners based on $J_{n \times n}$ achieve a substantial reduction (by an order of magnitude or better) in the number of iterations. As can be expected $J_{5 \times 5}$ performs better than $J_{3 \times 3}$, but will take more memory resources and computational time to implement. There is not a substantial loss in speedup when the incomplete $I L U(0)$ factorizations are used instead of the full inverse $J_{n \times n}^{-1}$. Therefore, in view of the additional memory resources (due to the filling of the sparse Jacobian) and CPU time that would be involved, more complete factorizations $I L U(k), k>0$, are not considered necessary.

\section{NUMERICAL RESULTS}

In this section we demonstrate the performance of the $I L U(0)-J_{n \times n}$ preconditioners for one of the test problems considered in Reference [5], in which a hydraulic fracture passes through a confining stress jump (refer to that paper for a complete description of the material properties and simulation parameters). This confining stress jump problem was chosen as it involves a substantial pinched region within which all the elements are subject to a width constraint, which is known to adversely affect the conditioning of the system. All the CPU times provided in this section were measured on a $2 \mathrm{GHz}$ Pentium IV computer under Windows 2000 Professional using the Compaq Visual Fortran compiler version 6.1.

In Figure 4(a) we plot the cumulative CPU times spent by the BiCGSTAB algorithm with diagonal preconditioning compared to that of the BiCGSTABILU $3 \times 3$ and $5 \times 5$ algorithms versus the number of active elements $N$ in the fracture. The variation in the number of active elements is obtained by considering the solution times associated with the increasing fracture footprints measured over the evolution the fracture. For any particular $N$, the data represent the cumulative CPU time of many consecutive solutions of the linear equations (7) associated with the implementation of Newton's method. We observe that the preconditioning based on the $I L U(0)$ factorization of $J_{n \times n}$ leads to considerably shorter solution times in spite of the fact that there is a significant region within which there are active width constraints. In Figure 4(b) we plot the cumulative speedup ratio for the $I L U(0)-J_{n \times n}$ preconditioners. We observe that the current algorithm delivers more than a 10-fold speedup for the relatively moderate number of active elements $N=1600$ and compares very favorably with the MG preconditioners (see Reference [5]), which achieved a cumulative speedup of about 6. It is important to note that the $J_{n \times n}$ yield dividends almost immediately $(N \gtrsim 100)$, which is in contrast to the MG preconditioners which only start to be more efficient beyond $N \approx 400$ active elements.

In Table I we provide a sample of iteration counts and CPU solution times for this confining stress jump problem. We observe that the iteration counts for the BiCGSTAB algorithm with diagonal preconditioning exhibit a power law increase in the number of iterations $\left(\propto N^{2 / 3}\right)$, the MG preconditioner has a relatively constant iteration count, and the $J_{n \times n}$ preconditioners exhibit a small increase in the number of iterations. Though the MG preconditioners achieve a constant iteration count, the cost per iteration is almost double that of the localized Jacobian preconditioners considered here. The MG preconditioners achieve a speedup ratio of approximately 6, while the localized Jacobian preconditioners achieve speedup ratios of close to 12 .

For other cases that have been simulated, (for which the results are not presented here since the trends are the same) the speedup factors due to the $I L U(0)-J_{n \times n}$ preconditioners grow 

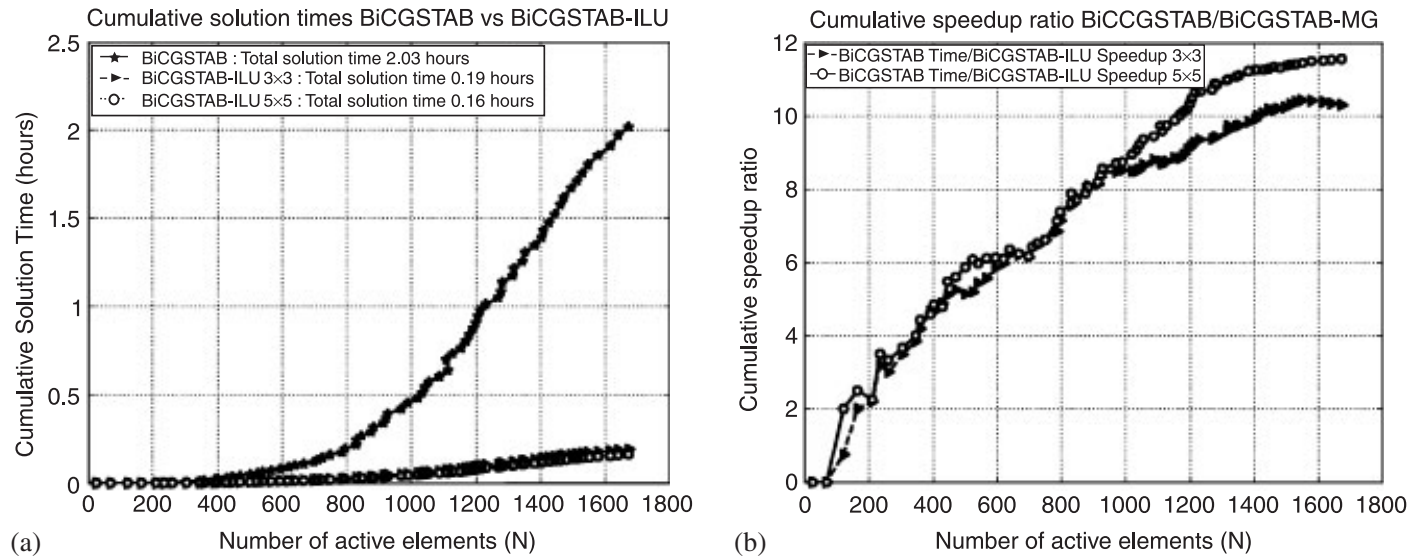

Figure 4. (a) Cumulative CPU times spent on the solution process by the BiCGSTAB $(\star)$ and the BiCGSTABILU algorithms ( $\downarrow$ for $J_{3 \times 3}$ ) and (o for $J_{5 \times 5}$ ); and (b) the cumulative speedup ratio ( $\downarrow$ for $\left.J_{3 \times 3}\right)$ and $\left(\circ\right.$ for $\left.J_{5 \times 5}\right)$ as measured by $T_{\text {BiCGSTAB }} / T_{\text {BiCGSTABILU }}$.

Table I. Comparative average iteration counts and CPU times for Jacobian inversion for a fluid driven fracture in a medium with a 6.6-0 MPa confining stress jump using BiCGSTAB with Diagonal, MG $V(0,2)$, and $I L U(0)-J_{n \times n}$ preconditioning.

\begin{tabular}{|c|c|c|c|c|c|c|c|c|}
\hline & \multicolumn{2}{|c|}{ Diagonal } & \multicolumn{2}{|c|}{$V(0,2)$ MG (see Reference [5]) } & \multicolumn{2}{|c|}{$I L U(0)-J_{3 \times 3}$} & \multicolumn{2}{|c|}{$I L U(0)-J_{5 \times 5}$} \\
\hline & Iterations & CPU (s) & Iterations & CPU (s) & Iterations & CPU (s) & Iterations & CPU (s) \\
\hline 104 & 34 & 0.02 & 8 & 0.07 & 7 & 0.01 & 5 & $<0.01$ \\
\hline 257 & 57 & 0.05 & 8 & 0.08 & 8 & 0.01 & 6 & 0.01 \\
\hline 500 & 81 & 0.24 & 8 & 0.10 & 8 & 0.03 & 6 & 0.03 \\
\hline 749 & 94 & 0.41 & 7 & 0.11 & 9 & 0.05 & 6 & 0.05 \\
\hline 1001 & 120 & 1.05 & 8 & 0.16 & 10 & 0.10 & 7 & 0.09 \\
\hline 1252 & 130 & 1.30 & 7 & 0.20 & 10 & 0.13 & 7 & 0.11 \\
\hline 1595 & 148 & 1.92 & 8 & 0.25 & 11 & 0.16 & 7 & 0.14 \\
\hline
\end{tabular}

steadily with the number of elements, reaching values of close to 20 when $N \approx 5000$. The number of required preconditioned iterations increases as a power law (approximately $\propto N^{1 / 3}$ ) whereas the number of iterations for the standard BiCGSTAB algorithm increases as a power law $\propto N^{2 / 3}$. Asymptotically (for $N>1000$ ) the cost of performing a single BiCGSTAB iteration with $J_{3 \times 3}$ preconditioning is approximately 1.15 times that of performing a standard BiCGSTAB iteration, while the cost of performing a single BiCGSTAB iteration with $J_{5 \times 5}$ preconditioning is approximately 1.4 times that of a standard BiCGSTAB iteration. Thus although $J_{5 \times 5}$ manages to achieve a greater reduction in the number of iterations compared to $J_{3 \times 3}$, this gain is lost somewhat due to the additional CPU costs per iteration. 


\section{CONCLUSIONS}

In this paper, we have described a class of sparse localized approximations to the Jacobian of the coupled system of integro-partial differential equations that model the evolution of a fluid driven fracture. Exploiting the rapid $O\left(1 / r^{3}\right)$ decay of the Green's function kernel of the integral operator makes it possible to truncate the fully populated influence operator to yield a localized approximation to the Jacobian. Such localized Jacobians proved beneficial [5] in the MG context in which they were used to yield very effective iterative smoothers for damping the high-frequency components of the problem. Because the approximation to the Jacobian is localized, it is to be expected that the high-frequency modes of the full Jacobian are well captured. However, because of the rapid decay of the kernel, we see that the localized Jacobians also yield a great deal of information about the non-local properties of the Jacobian.

In this paper, we have investigated the spectral properties of these localized Jacobians both analytically using Fourier Transforms for a linearized 1D model problem and directly for a realistic hydraulic fracture problem. The model problem analysis enabled us to establish the potential of the class I localized Jacobians as efficient preconditioners, while it was possible to rule out the use of class II localized Jacobians. The direct numerical spectral analysis of the planar hydraulic fracture problem enabled us to demonstrate that the basic ILU(0) factorizations yield a sufficiently good preconditioner compared to the exact inverse that more complete factorizations $I L U(k), k>0$, with concomitant fill, are unnecessary. The concept of using $I L U$ factorizations of localized approximations of fully populated operators could be used in other contexts, for example preconditioners constructed from local approximations of singular integral operators with rapidly decaying kernels could achieve similar reductions in computational effort.

In a numerical example involving a fracture propagating through a confining stress jump, we have demonstrated that $I L U(0)$ factorizations of these localized Jacobians $J_{n \times n}$ yield an efficient class of preconditioners that can substantially reduce the number of iterations that are required to solve the Jacobian system. Indeed, the break-even point for the beneficial use of these preconditioners occurs very rapidly as $N$ is increased-for as little as 100 elements. The preconditioners yield 10-fold speedup of the solution process for problems with $N \approx 1500$ elements, which increases to factors as high as 20 for problems with $N \approx 5000$. The $J_{5 \times 5}$ preconditioners achieve lower iteration counts than the $J_{3 \times 3}$ preconditioners, but this gain is offset by the additional cost per iteration. Unlike MG preconditioners, for which the iteration counts remain essentially constant as the number of elements grows, the iteration counts for the $I L U(0)-J_{n \times n}$ preconditioners grow as a power law $\propto N^{1 / 3}$ with the number of elements. However, the cost per iteration of implementing the $I L U(0)-J_{n \times n}$ preconditioners is substantially less than that of MG, which is at best a factor of 2 .

\section{ACKNOWLEDGEMENTS}

The author would like to acknowledge the support of NSERC for his research program.

\section{REFERENCES}

1. Clifton RJ, Abou-Sayed AS. On the Computation of the Three-Dimensional Geometry of Hydraulic Fractures, SPE 7993, Presented at the SPE/DOE Symposium of Low-Permeability Gas Reservoirs, Denver, CO, 1979. 
2. Clifton JR. Three-Dimensional Hydraulic Fracturing. SPE Monograph, 1989.

3. Peirce AP, Siebrits E. Uniform asymptotic approximations for accurate modelling of cracks in layered elastic media. International Journal of Fracture 2001; 110:205-239.

4. Peirce AP, Siebrits E. The scaled flexibility matrix method for the efficient solution of boundary value problems in 2D and 3D layered elastic media. Computer Methods in Applied Mechanics and Engineering 2001; 190:5935-5956.

5. Peirce AP, Siebrits E. A dual mesh multigrid preconditioner for the efficient solution of hydraulically driven fracture problems. International Journal for Numerical Methods in Engineering 2005; 63:1797-1823.

6. Axelsson O. Iterative Solution Methods. Cambridge University Press: Cambridge, 1996.

7. Quarteroni A, Sacco R, Saleri F. Numerical Mathematics. Springer: New York, 2000.

8. van der Vorst H. BiCGSTAB: a fast and smoothly converging variant of Bi-CG for the solution of nonsymmetric linear systems. SIAM Journal on Scientific and Statistical Computing 1992; 13:631-644.

9. Barrett R, Berry M, Chan TF, Demmel J, Donato J, Dongara J, Eijkhout V, Pozo R, Romine C, van der Vorst H. Templates for the Solution of Linear Systems: Building Blocks for Iterative Methods. SIAM: Philadelphia, 1994. 